\title{
TestDaF oder ZOP? \\ Welche Prüfung sagt mehr über die Studierfähigkeit aus?
}

\author{
Susanne Katharina Schleif (Leipzig)
}

\begin{abstract}
The following article is about two tests of the knowledge of German as a foreign language on an advanced level: TestDaF and ZOP (Zentrale Oberstufenprüfung, Goethe-Institut). Each test type is first described and then analysed for its suitability as a university entry test for foreign students, as both are recognised as such by German universities. It is shown that every test type includes items, which are not valid for university applicants. Based on this analysis, the author works out criteria, which a university entry test should include.
\end{abstract}

\section{$1 \quad$ Einleitung}

TestDaF (Test Deutsch als Fremdsprache) ist die Deutschprüfung der letzten Jahre, aber was zeichnet erfolgreiche TestDaF-Absolventen aus? Inwieweit bestehen qualitative Unterschiede zu Absolventen anderer Deutschprüfungen des Oberstufenniveaus?

Im folgenden Artikel sollen zunächst die Prüfungsformate von TestDaF und ZOP (Zentrale Oberstufenprüfung des Goethe-Instituts) beschrieben und daraus die Kompetenzen abgeleitet werden, die die Lerner in den jeweiligen Vorbereitungskursen erwerben. Sodann wird auf die Schwachpunkte der jeweiligen Prüfung eingegangen. Zusammenfassend werden die Kompetenzen dargestellt, die ein (ausländischer) Studienbewerber braucht, um an einer deutschen Universität studieren zu können. Die DSH (Deutsche Sprachprüfung für den Hochschulzugang ausländischer Studienbewerber) bleibt hier unberücksichtigt, weil diese Prüfung hinsichtlich Testformat, Schwerpunkten und Bewertungsvorgaben nicht einheitlich ist und die Ergebnisse daher nicht vergleichbar sind. Es soll daran erinnert werden, dass TestDaF konzipiert wurde, um über eine in Aus- und Inland einheitliche Prüfung für den Hochschulzugang zu verfügen.

Ich unterrichte seit 4 Jahren an der VHS Leipzig, zunächst im Grundstufenbereich, seit 2002 auch in Mittel- und Oberstufenkursen für die Vorbereitung von ZMP, TestDaF und ZOP. Wir haben an der VHS im Kollegenteam differenzierte Lehrpläne entwickelt, die intensiv auf die jeweilige Prüfung vorbereiten. Sowohl Kursleiter als auch Lerner beurteilen die beiden Oberstufen-Module sehr unterschiedlich. Der vorliegende Artikel wird dafür eine Reihe von 


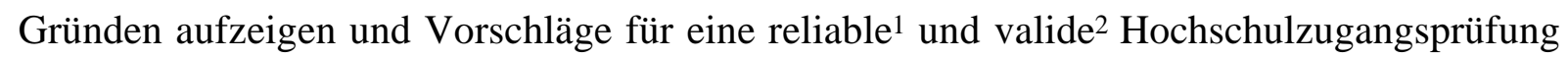
unterbreiten.

\section{Beschreibung der Prüfungen TestDaF/ ZOP}

\section{$\underline{\text { TestDaF }}$}

TestDaF ist ein standardisierter, zentraler und weltweit durchgeführter Sprachtest, der als Nachweis deutscher Sprachkenntnisse auf fortgeschrittenem Niveau dient und bei erfolgreichem Bestehen zur Studienaufnahme in Deutschland berechtigt. Dabei wird er als gleichwertig zur DSH angesehen (vgl. www.testdaf.de).

TestDaF ist ein Kompetenztest, d.h. es wird überprüft, inwieweit der Testkandidat die deutsche Sprache adäquat anwenden kann, nicht aber, welches Wissen er über die deutsche Sprache erworben hat. Da es um das Überprüfen von Fertigkeiten geht, ist es unerheblich, ob der Testteilnehmer zuvor einen Kurs besucht hat und welcher Art dieser Kurs war. Die Bewertung lehnt sich an die Kompetenzbeschreibungen des Europäischen Referenzrahmens ${ }^{3}$ an. Das Niveau TDN 4, ${ }^{4}$ mit dem seit dem WS 2004/05 die Zulassung zum Fachstudium an einer deutschen Universität gegeben ist (manche Universitäten/Fachbereiche akzeptieren auch TDN 4), entspricht dem Niveau C1.2, d.h. effektive kompetente Sprachanwendung. ${ }^{5}$

Die sprachliche Grundlage des Tests ist die Allgemeinsprache. Es fließen allerdings Elemente der Fachsprache ein. Alle Testaufgaben antizipieren Kommunikationssituationen, die sich auf den Studentenalltag sowie das Fachstudium an einer deutschen Universität beziehen.

\footnotetext{
${ }^{1}$ Die Reliabilität beschreibt die Zuverlässigkeit eines Tests, d.h. sind die Ergebnisse unabhängig von Ort, Zeit und Prüfer, sind Lernerleistungen vergleichbar und reproduzierbar? Die Reliabilität ist hoch, wenn in einen Test verschiedene Aufgabenformate und Texte zu unterschiedlichen Themen einbezogen werden und jeder Lerner die gleichen Aufgaben mit klaren Instruktionen bearbeitet. Die Bewertung sollte objektiven bzw. objektivierten subjektiven Methoden folgen, mündliche Leistungen sind zur wiederholten Bewertung auf Kassette oder Video aufzunehmen.

2 Die Validität beschreibt die Relevanz eines Tests für eine bestimmte Zielgruppe, in unserem Fall also ausländische Studienbewerber. Es wird untersucht, ob im Test die Fertigkeiten und Kenntnisse überprüft werden, die laut Testbeschreibung überprüft werden sollten.

${ }^{3}$ Die Richtlinien des Europäischen Referenzrahmens teilen die Sprachkompetenz folgendermaßen ein: Niveau A = elementare Sprachverwendung, Niveau B = selbständige Sprachverwendung (B1 entspricht dem Zertifikat DaF, B2 beschreibt die untere Mittelstufe), Niveau C = kompetente Sprachverwendung (C1 entspricht etwa der Zentralen Mittelstufenprüfung, C2 der Zentralen Oberstufenprüfung).

${ }^{4}$ Das System des Europäischen Referenzrahmens kann je nach Lern- und Testsituation verfeinert werden. Für die Bewertung der TestDaF-Prüfung wurde folgende Subliste erstellt: TDN 3 verweisen auf eine sprachliche Kompetenz auf beginnendem/ mittlerem Mittelstufenniveau (B2.1., B2.2.), TDN 4 entspricht dem Niveau C1.1. (abschließende Mittelstufe), TDN 5 entspricht schließlich dem Niveau C1.2., der beginnenden Oberstufe. Dies erklärt die Ergebnisvorgaben der jeweiligen Universitäten, die darauf achten, dass TestDaF den anderen zulassenden Deutschprüfungen vom Schwierigkeitsgrad her in etwa entspricht.

5 Das Oberniveau C1 (Effective Operational Proficiency) wird folgendermaßen beschrieben: Ein Lerner auf C1Niveau sollte adäquat in beruflichen Kontexten agieren können, flüssig und spontan kommunizieren, über einen großen Wortschatz verfügen, aber trotzdem paraphrasieren können, verfeinerte Diskursstrategien anwenden können und schriftliche und mündliche Redebeiträge logisch strukturieren (vgl. Europarat 2001).
} 
TestDaF wird von allen Testkandidaten komplett durchgeführt. Bei der Bewertung wird ein Profil erstellt, woraus die Leistungen in den einzelnen Teilbereichen ersichtlich werden.

TestDaF setzt sich aus folgenden Prüfungsteilen zusammen:

- Leseverstehen (LV), 60 min
LV 1:
Zuordnen von Kurztexten
LV 2:
Auswahlantworten $(\mathrm{a} / \mathrm{b} / \mathrm{c})$
LV 3:
Alternativantworten (ja/ nein/ nicht im Text)

- Hörverstehen (HV), 40 min

HV 1: $\quad$ Kurzantworten zu einem Telefonat, Studentenalltag

HV 2: $\quad$ Alternativantworten zu einem Interview/Vortrag (ja/nein)

(einmal)

HV 3: $\quad$ Kurzantworten zu einem Interview/Vortrag

(zweimal)

- Schriftlicher Ausdruck (SA), 60 min

- Verfassen einer Einleitung, Vorstellen der Gliederung

- Beschreiben einer Statistik

- Wiedergeben fremder Meinungen/Stellungnahmen

- Verfassen einer eigenen Stellungnahme zum Thema

- Mündlicher Ausdruck (MA), 30 min
MA 1:
Vortragen eines Anliegens
MA 2:
Geben und Erfragen von Informationen
(4 Aufgaben)
MA 3:
Beschreiben einer Graphik und einer Statistik
(2 Aufgaben)
MA 4:
(3 Aufgaben)
Begründen von Entscheidungen, Erwägen von Vor- und
Nachteilen

Der Test enthält also einige Aufgaben, die valide und authentisch für die Zielgruppe, also ausländische Studenten und Wissenschaftler, sind. Dazu zählen LV1, wo es um globales Lesen und Zuordnungen geht, HV1/3, wo Kurzantworten zu Hörtexten aus dem Studentenalltag und einem Vortrag elizitiert werden sowie MA1/2/4, wo die Studenten in authentischen Minisituationen des Studentenalltags ${ }^{6}$ agieren sollen.

TestDaF zeichnet sich durch eine sehr hohe Reliabilität aus, d.h. die Testergebnisse sind unabhängig von örtlichen und zeitlichen Gegebenheiten sowie den Testleitern. Hör- und Lesetexte beziehen sich auf unterschiedliche Themen, es werden unterschiedliche Aufgabenformate angeboten. Die Schreibaufgabe ist durch Vorgaben so gestaltet, dass jeder Testkandi-

\footnotetext{
${ }^{6}$ Als problematisch erscheint mir die Präsentation der Mini-Situationen, die zwar Authentizität vorgeben, durch die verwendeten Namen und Hintergründe aber nicht authentisch sind (z.B. Der Seminarleiter, Herr Dr. LasisZimuso, fragt Sie, was Sie dazu meinen ..., Fabouda, 2002, S. 143). Auf der Prüferschulung zu einem ähnlichen Test (SOPI - Simulated Oral Proficiency Interview, kassettenbasierter mündlicher Test, der sich auf die ACTFLKompetenzrichtlinien bezieht; vgl. www.cal.org), wurden die Teilnehmer aufgefordert, möglichst neutrale Situationen auszuformulieren, in die sich die Testteilnehmer besser einfühlen und eindenken können.
} 
dat auf die gleichen Informationen zurückgreifen kann. Der mündliche Subtest basiert auf Kassetten und umfasst verschiedene Subfertigkeiten und Themenkontexte, die nicht alle durch einen direkten Test, z.B. ein Prüfungsgespräch, abgedeckt werden könnten.

$\underline{\mathrm{ZOP}}$

Die ZOP ist ein Deutschtest der Oberstufe und weist differenzierte Kenntnisse sowie eine qualifizierte Beherrschung der deutschen Sprache im Berufsleben nach. Die Testteilnehmer sollen sich mündlich und schriftlich gewählt ausdrücken können (vgl. www.goethe.de). Ein erfolgreiches Bestehen befreit von der Teilnahme an der DSH und berechtigt zur Aufnahme eines Studiums an einer deutschen Universität.

Die ZOP ist überwiegend ein Leistungstest, d.h. es werden die in einem vorbereitenden Sprachkurs erworbenen Sprachkenntnisse und -fertigkeiten überprüft, dabei werden ca. 1.200 UE vorausgesetzt.

Sprachliche Grundlage der ZOP ist die Standardsprache. Der Textkorpus setzt sich zumeist aus anspruchsvollen journalistischen Texten sowie allgemeinwissenschaftlichen Abhandlungen zusammen.

Kritisch ist, dass an einigen Goethe-Instituten die ZOP an mehreren Tagen durchgeführt wird und die Lerner trotz Bezahlung des Komplettpreises nach dem Scheitern in einer Teilprüfung nicht die Möglichkeit bekommen, die anderen Subtests wenigstens auszuprobieren.

Die ZOP setzt sich aus folgenden Prüfungsteilen zusammen:

- Texterklärung, 90 min

- Beantworten von Fragen zu einem langen Lesetext in ganzen Sätzen

- Umformen von Textstellen

- Vornehmen von Paraphrasierungen/Einsetzen von Synonymen

- Ausdrucksfähigkeit, 90 min

- Einsetz-, Transformations- und Ergänzungsübungen zu Aspekten der

Oberstufengrammatik

- Hörverstehen, 30-40 min

- Stichpunktartiges Beantworten von Fragen zu einem langen Hörtext

- Schriftlicher Ausdruck, 90 min

- Verfassen eines Aufsatzes zu einem literarischen oder freien Thema

- Mündlicher Ausdruck

- Halten eines vorbereiteten Vortrags zu einem selbst gewählten Thema

- Diskutieren über das im Vortrag angesprochene Thema

- Lautes Vorlesen eines Lesetextes und mündliche Zusammenfassung

In den ZOP-Subtests "Textverstehen" und "Ausdrucksfähigkeit" werden direkt die Sprachkenntnisse, also Wortschatz und Grammatik, auf Oberstufenniveau getestet. Es werden Aufgaben zu Paraphrasierungen, Synonymen/Antonymen, Transformationen und Umformulierungen gestellt. In diesen Subtests wird überprüft, inwieweit der Testteilnehmer das System der deutschen Sprache verstanden hat und über eine gewisse sprachliche Leichtigkeit verfügt, um Textabschnitte zu variieren und zu nuancieren. 
Die Validität der ZOP für Studienanwärter in Deutschland ist sehr hoch. So stellt das Beantworten von Fragen zu einem journalistischen Text/Essay eine authentische Handlung im Studium dar. Bei den Aufgaben zum Hörverstehen sind entsprechend der authentischen Anforderungen im Studium aussagekräftige Notizen zu erstellen. Die Aufgaben der mündlichen Prüfung sind - von der Art der Vorbereitung abgesehen - dem studentischen Alltag angemessen: mündliches Zusammenfassen eines Lesetextes, spontane Diskussionen sowie das Halten von Vorträgen. Wenn es in einem Sprachtest für Studienbewerber als wichtig erachtet wird, dass die Testteilnehmer sowohl über sprachliches als auch inhaltliches Wissen verfügen, dann ist die Aufgabenstellung für den Aufsatz der ZOP angemessen, denn hier geht es um die Präsentation und Strukturierung von Ideen und Kenntnissen zu einem Thema. Der ZOP-Aufsatz stellt eine eigenständige geistige Leistung dar.

Die Reliabilität der ZOP ist jedoch nicht so hoch wie die des TestDaF, da viele Aufgabenformate frei sind und eine eindeutige Bewertung somit nicht gegeben ist. So werden für den Aufsatz literaturbezogene und freie Themen ohne Vorgaben präsentiert. Beim LV müssen die Studenten in ganzen Sätzen antworten, was nicht der Realität studentischer Leseanforderungen entspricht. Außerdem ist nicht eindeutig, worauf sich sodann die Bewertung bezieht, auf die herausgelesenen Informationen oder die Schreibfertigkeit. Die Subtests der mündlichen Prüfung können entweder zu Hause (Vortrag) oder vor der Prüfung vorbereitet werden.

Hinsichtlich der Textauswahl ist Folgendes zu bemerken: In der TestDaF-Prüfung wird thematisch auf die Hochschulbezogenheit geachtet, d.h. die Texte und Aufgaben beziehen sich auf relevante Kontexte aus dem Hochschulalltag, aber auch aus dem Fachstudium. Die Themenkomplexe sind u.a. Umwelt, Naturwissenschaften, Wirtschaft/Technik, Psychologie und Sprachwissenschaft. Dabei ergeben sich natürlich Schwierigkeiten hinsichtlich der Spezialisierung der Studienbewerber. Aus diesem Grund wäre es angemessen, entweder äquivalente Prüfungsformate TestDaF für Naturwissenschaftler und für Geisteswissenschaftler zu erarbeiten oder sich inhaltlich auf Themen des allgemeinen Interesses festzulegen. Auch in ZOP-Texten sollte darauf geachtet werden, dass Texte von allgemeinem Interesse auf einer angemessenen Sprachebene gewählt werden, mit denen vorrangig die Sprachkompetenz gemessen wird und nicht ein bestimmtes Hintergrundwissen. ${ }^{7}$

\section{$3 \quad$ Kompetenzen der Kursteilnehmer}

Ausgehend von den Schwerpunkten des jeweiligen Prüfungsformats sowie eigenen Eindrücken von Lernerleistungen, Selbstanalysen von Lernern, Erfahrungsberichten von Lernern, die an beiden Oberstufenkursen der VHS Leipzig teilgenommen haben sowie Gesprächen mit anderen Kursleitern können bei Lernern aus TestDaF- bzw. ZOP-Kursen folgende Stärken diagnostiziert werden:

Teilnehmer von TestDaF-Kursen sind tendenziell gute Rezipienten sowohl von Lese- als auch Hörtexten mit fachlichem Hintergrund. Da aber im Unterricht nicht zwingend an einer

\footnotetext{
${ }^{7}$ Diese Analyse der Texte ist offensichtlich bei der Erstellung des ZOP-Übungssatzes 03 (Kaspar Hauser) auch nicht durchgeführt worden, denn dieser enthält z.T. ein etwas altmodisches Deutsch.
} 
qualitativen Verbesserung der produktiven Fertigkeiten gearbeitet werden muss, ${ }^{8}$ sind sie eher mittelmäßige Produzenten. Da durch das Fehlen eines Prüfungsteils zu den Sprachkenntnissen der Druck und im Sinne des Aspekts der negativen Rückwirkung ${ }^{9}$ auch die Motivation fehlt, sich auch zu Hause intensiv mit strukturellen Merkmalen der Sprache auseinandersetzen, fehlen den Lernern strukturelle Festigkeit und die sprachliche Selbstkontrolle (Monitoring). Die Lerner tun sich schwer, Umformulierungen vorzunehmen, Akzente und Nuancen zu verstehen und selbst zu setzen und oft auch Referate und Hausarbeiten angemessen zu verfassen. All das sind Aspekte, die für ein Studium in Deutschland wichtig sind. Dennoch sind bei den TestDaF-Lernern Kommunikations- und Kompensationsstrategien gut ausgeprägt. Das kann damit erklärt werden, dass die erworbenen Kenntnisse so effektiv wie möglich eingesetzt werden, unbekannte Wörter werden umschrieben, komplizierte Strukturen vereinfacht.

Bei Teilnehmern an ZOP-Kursen ist das System der deutschen Sprache besser integriert, sie verstehen den Zusammenhang zwischen Inhalt und Form besser und können eigene Texte vielseitiger gestalten. Sie sind gute Produzenten, manchmal ist jedoch durch den verspürten Leistungsdruck der Prüfung eine eingeschränkte Spontaneität bei übermäßiger Selbstkontrolle festzustellen. Das Curriculum ist sehr strukturlastig, so dass es zu einer Vernachlässigung inhaltlicher Aspekte bei der Rezeption kommen kann. Die Lerner werden durch die detaillierten Aufgaben zum Lesetext dazu angehalten, den Text möglichst so genau wie möglich zu erschließen. Das bedeutet natürlich die Vernachlässigung global orientierter Erschließungstechniken, die im Studium aufgrund der dort zu bewältigenden Textlängen aber wichtig wären.

Wenn ein Sprachkurs nicht nur als effektive Prüfungsvorbereitung angesehen wird, sondern in erster Linie an der Verbesserung der Sprachkompetenz der jeweiligen Zielgruppe gearbeitet werden soll, dann schneiden TestDaF-Lerner schlechter ab als ZOP-Lerner. Im TestDaFUnterricht muss viel Unterrichtszeit für das Üben der verschiedenen Aufgabenformate investiert werden, was mehr mit der Entwicklung der logischen als der sprachlichen Fertigkeiten zu hat. Aufgrund der Entwicklung von Testknackerstrategien können Prüfungsergebnisse nur selten eindeutig interpretiert werden. Im ZOP-Kurs verläuft das Vermitteln und Üben der lexikalischen und grammatischen Mittel mit der Anwendung in Testaufgaben synchroner.

\footnotetext{
${ }^{8}$ Im Sinne der Anforderungen an die Prüfungsteile Ausdrucksfähigkeit und Textverstehen der ZOP.

${ }^{9}$ Mit Rückwirkung wird in der Testwissenschaft der Einfluss eines Tests auf das vorbereitende Lehren und Lernen beschrieben, wie verändert sich die Lehrpraxis hinsichtlich Validität und Authentizität? Hinsichtlich von TestDaF ist einmal interessanterweise festzustellen, dass das Fehlen eines strukturellen Prüfungsteils auch zu einer negativen Rückwirkung führen kann. Ich würde das mit einem Kochen im DaF-Grundstufensaft beschreiben.
} 


\section{Schwächen der Prüfungskonzepte und Aufgabenformate}

Ausgehend von den im vorherigen Abschnitt beschriebenen Kompetenzen der Lerner sowie einer Aufgabenanalyse hinsichtlich Validität und Reliabilität sollen die Schwächen der Prüfungskonzepte aufgeführt und mit Beispielen belegt werden:

\section{$\underline{\text { TestDaF }}$}

LV und HV sind Fertigkeiten, die nur indirekt getestet werden können, aber die Aufgaben müssen der Realität angemessen sein. Die Aufgaben LV2/LV3/HV2 sind zwar leicht und reliabel auszuwerten, aber nicht valide. Außerdem hat sich herausgestellt, dass die Angabe "nicht im Text" beim LV3 nicht eindeutig definiert ist und die Lerner verwirrt: Bedeutet "nicht im Text" gar nicht im Text oder so nicht im Text? Was ist mit eindeutig korrekten oder eindeutig falschen Teilsätzen innerhalb einer uneindeutigen allgemeinen Aussage?

Bei den Aufgaben MA3/SA besteht meines Erachtens eine Statistik-/Graphiklastigkeit, die es in einem normalen geisteswissenschaftlichen Studium in dieser Form nicht gibt. Das Versprachlichen einer technischen Graphik ist für einen großen Teil der Zielgruppe nicht relevant und oft auch für gebildete Muttersprachler schwierig. Beim SA geht es anhand der verschiedenen Vorgaben um ein rein mechanisches Überprüfen von Subfertigkeiten der Fertigkeit Schreiben. Die Aufgabenstellung zum Teiltest SA ist sehr ausführlich, so dass es neben der Überprüfung der Fertigkeit SA in gewisser Weise auch um eine Überprüfung der Lesefertigkeit geht:

Modelltest SA, 60 min (Fabouda, 2002, S. 138)

Einleitung zum Thema Tourismus gegeben, darin einige Argumente/ relevanter Wortschatz für das Thema:

\section{Aufgabenstellung: Schreiben Sie einen Text zum Thema "Tourismus"}

- Graphikbeschreibung: dazu werden die wichtigsten Gesichtspunkte schon ge-

nannt: Anteil der Urlaubsreisenden, Verhältnis von Inlands- und Auslandsreisen

- Wiedergabe von zwei vorgegebenen Stellungnahmen

- Verfassen der eigenen Meinung, Vergleich mit dem Heimatland

Erster Kritikpunkt ist die Zeitvorgabe von $60 \mathrm{~min}$, in denen alle 3 Aufgaben erfüllt und zusätzlich eine Einleitung verfasst werden sollen. Da TestDaF aber ein Test ist, in dem Deutschlerner ihre Studienfähigkeit nachweisen sollen, können die kognitiven Fertigkeiten, also wie Lerner ein Thema aufbauen und wie sie argumentieren, nicht vernachlässigt werden. Außerdem werden manchmal für Testkandidaten im Ausland irrelevante Themen präsentiert, zu denen sie (noch) keinen Bezug haben ${ }^{10}$ und daher auch nichts darüber schreiben können. Vielleicht wäre hierbei wieder eine Unterteilung eines Formats TestDaF im Inland sowie TestDaF im Ausland sinnvoll oder die Präsentation eines Themas von allgemeinem Interesse.

10 Z.B. die Vor-/ Nachteile des Semestertickets an verschiedenen deutschen Universitäten diskutieren (TestDaF, Sommer 2003). 
Außerdem sehe ich - wie schon weiter oben erwähnt - ein Problem in der Vernachlässigung der strukturellen Aspekte und der daraus resultierenden mangelnden Motivation für die Entwicklung besserer textueller Kompetenzen, darunter die Stilistik.

\section{$\underline{\text { ZOP }}$}

Als großes Problem hinsichtlich der Reliabilität sehe ich an, dass die Aufgaben der Prüfungsteile "Textverstehen" sowie "Ausdrucksfähigkeit" thematisch miteinander zusammenhängen. Somit besteht nur eine geringe Möglichkeit eines Neustarts bei Schwierigkeiten mit dem Lesetext. Außerdem differenziert je nach Textschwierigkeit die Schwierigkeit der Umformungsübung:

Übungssatz 04, Texterklärung, Aufgabe 2:

Bitte ersetzen Sie die unterstrichenen Ausdrücke mit eigenen Worten nach ihrer Bedeutung im Text, und nehmen Sie, falls nötig, die sich daraus ergebenden Umformungen vor:

1... lassen es als unabweisbare Notwendigkeit erscheinen ...

Problematisch ist auch, dass der Hörtext beim 2. Mal in Abschnitten gehört werden darf. Die Frage stellt sich, ob zweimaliges Hören überhaupt einen validen Hörtest darstellt. Beim SA werden Wahlthemen präsentiert, was für den einzelnen Lerner mit dessen thematischen Vorlieben angenehm sein mag; die Vergleichbarkeit der Lernerleistungen ist dann aber nicht mehr gegeben:

Übungssatz 04, Freie Aufsatzthemen (250 Wörter)

Viele Menschen gehen heute aus beruflichen Gründen für eine kürzere oder längere Zeit ins Ausland. Was könnten wohl ihre Motive sein? ...

In der Boulevard-Presse wird sehr viel und oft sensationell über das Privatleben von Prominenten berichtet. Welche Gründe gibt es Ihrer Meinung nach für und gegen diese Art von Publikationen?

Im Deutschen gibt es das Sprichwort "Reden ist Silber, Schweigen ist Gold." Erläutern Sie, was damit gemeint ist. Schreiben Sie auch, was Sie persönlich von diesem Sprichwort halten $\cdots$

"Rauchen gefährdet die Gesundheit" - die der Raucher, aber eben auch die der Nichtraucher, wird oft behauptet. Deswegen werden in Deutschland immer mehr "Nichtraucherzonen" eingeführt ... Wie stehen Sie zu dieser Entwicklung? ...

Bei der mündlichen Prüfung ist der Aspekt der Vorbereitung kritisch. Der Vortrag, der zu einem selbst gewählten Thema gehalten wird, kann zu Hause, aber auch im Unterricht 
vorbereitet, kontrolliert, wiederholt und auswendig gelernt werden. Die Qualität hängt somit nicht mehr allein vom Lerner, sondern vom Fleiß seines Lehrers und der Intensität der Vorbereitung ab. Von einigen Kursleitern wird es als kritisch angesehen, dass "lautes Vorlesen" Bestandteil der mündlichen Prüfung ist. Ich sehe es aber als sehr sinnvoll an, die Phonetik in die Prüfung mit einzubeziehen, denn das "Verstandenwerden" ist ein wichtiger Aspekt des Studierens in Deutschland.

\section{$5 \quad$ Was müssen Studienbewerber denn nun sprachlich können?}

Wie in den vorherigen Abschnitten festgestellt werden konnte, weisen beide Prüfungsformate Vor- und Nachteile auf. Es bleibt also zu hinterfragen, über welche Fertigkeiten und Kenntnisse Studienbewerber an deutschen Hochschulen verfügen müssen und inwieweit ein Mix beider Prüfungsformate (wenigstens im vorbereitenden Unterricht) sinnvoll wäre:

\section{Leseverstehen:}

Die Studienbewerber müssen Lesetexte verschiedenster Themen unter einem gewissen Zeitdruck semantisch erfassen können, dabei sind sowohl globales als auch selektives Erfassen wichtig. Ebenso sollten die Studienbewerber Pointen, Nuancen und die Stellung des Autors zum Thema erkennen und interpretieren können.

Im studentischen Alltag stellt das Zuordnen von Informationen eine authentische Aufgabe dar, z.B. welche Veranstaltung gehört zu welchem Lehrgebiet (TestDaF LV1). Eine weitere authentische Aufgabe zum LV wäre das stichpunktartige Beantworten von Fragen (ZOP, vereinfacht). Dabei sollten vorrangig die inhaltlichen Aspekte bewertet werden, also die erfassten Informationen sowie deren Strukturierung. Hierbei geht es nicht um ein Testen der Fertigkeit Schreiben, da beim Stichpunktmachen die Notationsstrategien viel wichtiger sind. Diese müssten natürlich im Unterricht vermittelt und geübt worden sein, damit sie im Test bewertet werden können. Eine weitere authentische Aufgabe wäre das schriftliche oder mündliche Zusammenfassen von Texten (ZOP, mündlich), wobei es um eine Bewertung der Inhalte (Hauptinformation erfasst?), der systematischen Präsentation, der verwendeten Redemittel sowie der sprachlichen Richtigkeit ginge. Somit wäre die Zusammenfassung eine integrative Testmöglichkeit der Sprachkompetenz und könnte keiner Fertigkeit eindeutig zugeordnet werden. Wichtige Fertigkeiten, die sich auf das Rezipieren von Texten beziehen, aber ebenso nicht eindeutig der Fertigkeit LV zugeordnet werden können, ist das Umformulieren von Textstellen (ZOP) und das Wiedergeben von fremden Stellungsnahmen (TestDaF), denn es ist - wie jeder Student weiß - in Hausarbeiten von enormer Wichtigkeit, fremde Gedanken mit eigenen Worten auszudrücken, um nicht ständig zitieren zu müssen.

\section{Hörverstehen:}

Genau wie beim LV ist auch hier ein größerer Schwerpunkt auf das Erstellen von Stichpunkten zu legen, deren Aussagekräftigkeit auch bewertet werden kann. Außerdem sollten die Hörtexte in der Regel einmal, bei längeren und schwierigen Texten (u.a. mit Nebengeräuschen, mehreren Sprechern) zweimal gehört werden. Verzichtet werden muss auf das Hören in Abschnitten, weil dies authentischen Hörtätigkeiten an der Universität (eine Vorlesung 
dauert 90min) widerspricht. Es sollten ausschließlich populärwissenschaftliche Texte mit akademischer Orientierung eingesetzt werden.

Authentische Aufgaben sind das Beantworten von Fragen (TestDaF, ZOP) und das Erstellen einer Zusammenfassung, dazu gilt das Gleiche wie für das LV. Verzichtet werden sollte auf die reliablen Formate Alternativantworten und Auswahlantworten, weil sie nicht authentischen Hörhandlungen entsprechen.

\section{Schreiben:}

Da es sich um einen Test für Studienbewerber handelt, muss die Subfertigkeit "Akademisches Schreiben" bewertet werden. Die Texte müssen sich demnach durch hohe organisatorische und textuelle Kompetenz (vgl. Bachmann, 1990) auszeichnen, d.h. die Inhalte sollen logisch angeordnet sein und sprachlich angemessen präsentiert werden. Der effektive Einsatz von Argumentationsstrategien ist zu beachten. Ein Schwerpunkt der Bewertung muss auch der Gesichtspunkt Stilistik sein, d.h. der strukturelle und lexikalische Variantenreichtum. Von allen Studenten sollte ein bestimmtes populärwissenschaftliches Thema bearbeitet werden. Das Thema sollte zwar kontextualisiert werden, von Vorgaben ist jedoch abzusehen, weil die zukünftigen Studenten lernen müssen, eigene Ideen zu präsentieren. Ebenso sollte auf graphische Stimuli verzichtet werden, weil das Beschreiben von Graphiken oder Statistiken nur eine spezialisierte Subfertigkeit der Fertigkeit Schreiben darstellt.

Mündlicher Ausdruck:

Hinsichtlich der Prüfung zum mündlichen Ausdruck können Aufgaben aus beiden Prüfungsformaten gewählt werden, wie das Halten eines (nicht zu Hause) vorbereiteten Referats (ZOP), das Führen von spontanen Diskussionen (TestDaF, ZOP) und das Reagieren in Minisituationen des Studentenalltags (TestDaF).

\section{Sprachkenntnisse:}

Ein Prüfungsteil zu den Kenntnisbereichen der Sprache ist angebracht und notwendig. Bei der eigenen Sprachproduktion wird auf die sprachlichen Mittel zurückgegriffen, die der Lerner problemlos beherrscht, oft wird deswegen der Weg des "geringsten Widerstands" gewählt. In einen Subtest zu den Sprachkenntnissen können verschiedene Strukturmöglichkeiten integriert werden. Bei der Bewertung ist hinsichtlich der Gewichtung zu beachten, welche Fehler Grundstufen- und welche Oberstufenfehler sind.

Der Weg der Transformationen, der in der ZOP gegangen wird, ist grundsätzlich richtig, da hier auch verschiedene Möglichkeiten zugelassen werden.

Die hier präsentierten Erkenntnisse entstammen meiner Unterrichtserfahrung. Ich möchte damit die Diskussion anregen, die zur Erstellung einer für ausländische Studienbewerber sowohl reliablen als auch validen Prüfung für den Hochschulzugang führen soll. Wie festgestellt werden konnte, weist TestDaF bezüglich seiner Validität, die ZOP bezüglich ihrer Reliabilität noch erhebliche Defizite auf, um eindeutige Aussagen zur sprachlichen Kompetenz von Studienbewerbern zu treffen. 


\section{Literaturangaben}

Alderson, Charles/Clapham, Caroline/Wall, Diane (1995): Language Test Construction and Evaluation. Cambridge.

Bachman, Lyle F. (1990): Fundamental Considerations in Language Testing. Oxford.

Bachman, Lyle F./Palmer, Adrian S. (1996): Language Testing in Practise. Oxford.

Buscha, Anne/Linthout, Gisela (2002): Das Obenrstufenbuch - Deutsch als Fremdsprache. Leipzig.

Europarat (2001): Gemeinsamer europäischer Referenzrahmen für Sprachen: lernen, lehren, beurteilen. Berlin.

Glaboniat, Manuela (1998): Kommunikatives Testen im Bereich DaF. Innsbruck.

Glienicke, Stefan/Katthagen, Klaus-Markus (2002): TestDaF - Kurs zur Prüfungsvorbereitung. München.

Goethe-Institut (ed.): Zentrale Oberstufenprüfung - Übungssätze 01,03,04. München.

Kniffka, Gabriele/Gutzat, Bärbel (2003): Training TestDaF - Material zur Prüfungsvorbereitung. Berlin.

Lodewick, Klaus (2002): TestDaF Training - Vorbereitung auf den Test Deutsch als Fremdsprache. Göttingen.

www.goethe-institut.de, Informationen zur ZOP.

www.testdaf.de, Informationen zum TestDaF. 